\title{
Light in the east
}

\author{
Plans for the Extreme Light Infrastructure in eastern Europe are soon to be complete, with the choice of a \\ fourth, and final, site for the facility.
}

Across Eastern Europe, an unusual project is taking shape. The Extreme Light Infrastructure (ELI) will be a flexible, multi-purpose facility, hosting a variety of light sources in different countries. Funded by the European Union to the tune of $€ 700$ million, and set to be operational in 2015, ELI will be a hub for fundamental and applied research - laser and particle-accelerator engineering, biology and medicine, X-ray and gamma-ray imaging as well as a promoter of technology transfer. It's an ambitious task, but ELI promises to become a research leader in fields including laser acceleration, vacuum physics, attosecond science and photonuclear physics.

Three of the four planned ELI sites have already been chosen. The first, in Prague, Czech Republic, will host a compact laserplasma accelerator, providing ultrashort beams of energetic particles $(10 \mathrm{GeV})$ and radiation (up to a few $\mathrm{MeV}$ ). At the second site in Szeged, Hungary, a femtosecond laser will take snapshots of attosecond-scale electron dynamics in atoms, molecules, plasmas and solids. Nuclear processes will be investigated using a 30 PW laser housed at the third site in Magurele, Romania.

The location of the fourth site - possibly adjacent to one of the existing sites, or even in another country - will be decided later this year. And this is the big one: this site will be home to the real star of the ELI programme, a $200 \mathrm{PW}$, ultraintense laser, which is expected to advance laser acceleration to the ultrarelativistic regime.

Hosting the state-of-the-art ELI light source is a prize valued more highly by Eastern European nations than it would be by any other EU state. Although it is more than 20 years since the fall of the Berlin Wall and of the 'iron curtain' that divided the continent, science in Eastern Europe is still struggling against insufficient funding, an outdated research infrastructure and the consequences of decades of 'brain drain'. EU grants and other attempts to promote the return to Eastern Europe of its scientists working abroad have done little to alleviate these problems.

Meanwhile, Eastern European scientists are finding better access to funding and research facilities through joining international collaborations. Eastern European countries are heavily involved both in projects at the DESY laboratory in Hamburg, Germany, and in the experiments running at the Large Hadron Collider at CERN, in Switzerland - Hungary, Bulgaria and the Czech and Slovak Republics having all become CERN member states in the 1990s, Romania currently a candidate for accession. The advantages of such collaborations are undeniable, although long periods working away from home may be less than desirable for the scientists.

ELI, however, will support the science communities of Eastern Europe by creating local opportunities. Moreover, it will bring large international collaborations into Eastern Europe. The fourth ELI site, hosting its highest-intensity light source, is expected to be ranked eventually among the world's leading laboratories - each of the candidate sites is no doubt conscious of the advantages that will confer. It will be a difficult decision, but ultimately one site must be chosen to shine.

\section{Everyone's a winner}

\section{The bid to host the world's largest, most sensitive radio telescope has ended in a tie.}

It was a well-fought battle. Contenders South Africa and Australia each undertook a tremendous nine-year campaign to host the internationally funded Square Kilometre Array (SKA) - a telescope comprising thousands of dishes with a collecting area spanning a square kilometre ${ }^{1}$. Before the decision in late May, both countries had even started developing precursor facilities, in what might be interpreted as attempts to better their odds.

And the result? Both proposals will be used, in a dual-site plan that will stretch the budget - and the possibilities of the endeavour. The eight African countries partnering South Africa's bid will support development of a midfrequency (200-500 MHz) array, optimized to investigate the nature of gravity. The Australasian effort will probe the history and composition of the universe (including dark matter and dark energy) by fielding the low-frequency (70-200 MHz) range, and surveying hundreds of millions of galaxies.
The split approach capitalizes on the existing investments of each nation, and plays to the sites' individual strengths.

The decision was, however, met with disappointment in South Africa, widely held as favourite to host the entire array. Minister of Science and Technology Naledi Pandor issued a statement ${ }^{2}$ pointing out that an independent committee had deemed the African site the better option, and suggesting that the decision was motivated by a desire "to be inclusive".

Indeed, a report compiled by experts forming the SKA Site Advisory Committee (SSAC) identifies South Africa as the preferred site ${ }^{3}$. Many proponents of Australia's bid had hoped that the nation's wealth and stability might tip the scales in their favour. But the SSAC report attributed $75 \%$ of determining factors to scientific and technical concerns, and only $2 \%$ to political and socioeconomic issues.

Although it seems that a dual-site option was initially explored in the spirit of diplomacy, the process made clear the scientific advantages associated with the split. The project's myriad scientific goals ${ }^{4}$ demand a frequency range that was always going to require construction of more than one type of antenna. The decision to isolate these technologies diminishes the chance that the planned survey component of the project will compromise SKA's midfrequency ambitions.

Much has been made of the economic implications of the dual-site plan in the wake of its announcement. And it's clear that having two sites will increase the cost of the SKA - but that may be a reasonable trade for what should be a scientific (and diplomatic) triumph.

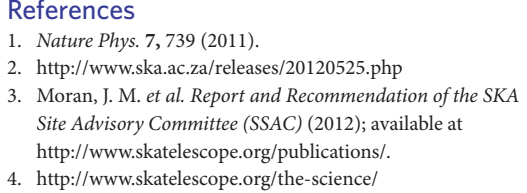

